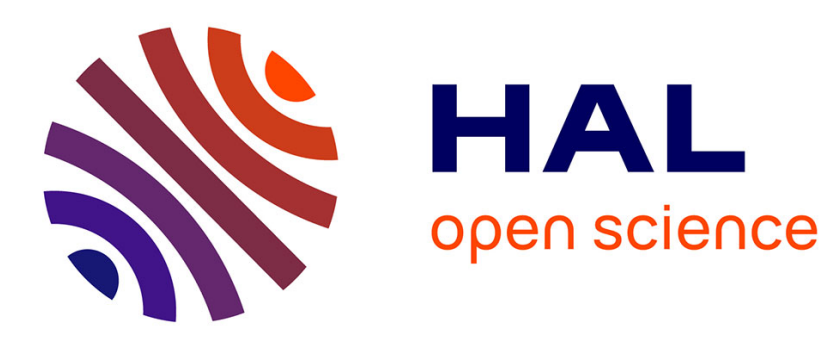

\title{
La controverse entre Gustav Landauer et le Vorwärts. Une querelle sur deux conceptions du socialisme
}

Anatole Lucet

\section{To cite this version:}

Anatole Lucet. La controverse entre Gustav Landauer et le Vorwärts. Une querelle sur deux conceptions du socialisme. Actuel Marx, 2020, 2020/2 (68), pp.156-174. 10.3917/amx.068.0156 . hal02949570

\section{HAL Id: hal-02949570 \\ https://hal.science/hal-02949570}

Submitted on 26 Sep 2020

HAL is a multi-disciplinary open access archive for the deposit and dissemination of scientific research documents, whether they are published or not. The documents may come from teaching and research institutions in France or abroad, or from public or private research centers.
L'archive ouverte pluridisciplinaire HAL, est destinée au dépôt et à la diffusion de documents scientifiques de niveau recherche, publiés ou non, émanant des établissements d'enseignement et de recherche français ou étrangers, des laboratoires publics ou privés. 


\title{
LA CONTROVERSE ENTRE GUSTAV LANDAUER ET LE VORWÄRTS. UNE QUERELLE SUR DEUX CONCEPTIONS DU SOCIALISME
}

\author{
Anatole LUCET
}

\author{
De toute l'histoire naturelle, je ne connais pas de créature plus \\ répugnante que le Parti social-démocrate. (Cris : Bravo!) $)^{l}$
}

Sur ce, je souhaite préciser que je considère indigne de moi de répondre dûment à cette attaque puérile d'un fanatique irresponsable. ${ }^{2}$

Remarquable, pas vrai? Tous deux, Marx et Landauer, veulent le socialisme. ${ }^{3}$

En près de trente années d'activisme, rien ne semble s'être perdu du mordant avec lequel le penseur et militant anarchiste Gustav Landauer (1870-1919) s'en prit à la social-démocratie marxiste de son temps ${ }^{4}$. Lorsqu'en 1890, profitant de l'abandon des «lois socialistes (Sozialistengesetze) » (un ensemble de lois initiées en 1878 par Otto von Bismarck pour interdire les organisations socialistes), le Parti social-démocrate allemand (SPD) se reforme et adopte le nom qu'on lui connait encore aujourd'hui, de vives dissensions internes se font jour. Rejouant pour partie des désaccords anciens sur lesquels s'était structuré le mouvement socialiste, ces tensions se muent en une controverse polyphonique entre la ligne du parti et les tendances divergentes. À la même période, le quotidien Vorwärts reprend sa parution, doté d'un nouveau sous-titre : l' « organe central du Parti social-démocrate allemand » est alors la voix officielle de l'orthodoxie marxiste .

L'affirmation d'August Bebel citée en exergue est à l'image des autres apparitions du nom de Gustav Landauer dans les pages du Vorwärts, qui le présente comme un être borné, fanatique, abscons et peu rigoureux. Les rédacteurs n'ont pas de mots assez cinglants pour dénigrer celui qui, dans son Appel au socialisme, qualifiera le marxisme comme « la peste de notre temps et la malédiction du mouvement socialiste $! »^{6}$. Qu'un auteur se soit acharné toute sa vie à dénoncer l'institution devenue en 1912 le parti majoritaire au Reichstag peut

\footnotetext{
${ }^{1}$ Gustav Landauer, discours devant le Congrès des conseils d'ouvriers, de paysans et de soldats bavarois, $1^{\text {er }}$ mars 1919.

${ }^{2}$ August Bebel, « Noch einmal Herr Gustav Landauer » (Encore monsieur Gustav Landauer), Vorwärts, 10, $\mathrm{n}^{\circ}$ 237, 8 octobre 1893 .

${ }^{3}$ Walter Trojan, Junge Menschen, III, n ${ }^{\circ}$ 7-8, avril 1922.

${ }^{4}$ Les publications de Gustav Landauer sont citées dans leur édition originale. Les textes réédités dans les Ausgewählte Schriften (édités par Siegbert Wolf, Lich/Hessen, Verlag Edition AV, 2008-2019), sont signalés par l'acronyme GLAS suivi du numéro du volume. La pagination indiquée suit alors cette édition. Sauf mention contraire, les traductions sont de moi. La Gustav Landauer Online Bibliography propose depuis 2016 une base de donnée complète et à jour des travaux de et sur Landauer (traductions comprises) : www.zotero.org/groups/gustav_landauer_online_bibliography

${ }^{5}$ Les archives du Vorwärts ont été intégralement numérisées et mises à disposition par la bibliothèque de la Friedrich-Ebert-Stiftung, depuis la fondation du journal en 1876 jusqu'à son interdiction en 1933 (https://www.fes.de/bibliothek/vorwaerts-blog/). Sur l'histoire du Vorwärts, cf. Schueler Hermann, Trotz alledem. Der Vorwärts - Chronist des anderen Deutschland, Berlin, Vorwärts Buch, 2006.

${ }^{6}$ Landauer Gustav, Appel au socialisme (1911), Angaut Jean-Christophe et Lucet Anatole (trad.), Saint-Michelde-Vax, La Lenteur, 2019, p. 34.
} 
s'expliquer de diverses façons: le Vorwärts pencha pour une série d'interprétations psychologisantes présentant l'auteur anarchiste sous un jour peu reluisant. Parallèlement, le journal Der Sozialist dirigé par Landauer n'eut de cesse de décrier les dirigeants du SPD. Cet article part des multiples références à Gustav Landauer dans le Vorwärts pour déceler, derrière la violence de la polémique, les grandes lignes d'une controverse théorique fondamentale sur la conception du socialisme et du changement social. Cette analyse permettra de comprendre, en dépit du mépris affiché par Bebel, la récurrence du nom de Landauer dans les colonnes du journal officiel de la social-démocratie allemande.

\section{« L'INÉVITABLE MONSIEUR LANDAUER ! »}

Celui que l'on fit passer, depuis sa première mention dans les pages du Vorwärts jusqu'après sa mort, pour un artiste agité ou pour un agitateur inoffensif, était pourtant considéré par la police de son temps comme «l'agitateur le plus important du mouvement révolutionnaire radical [...] dans $1^{\prime}$ Allemagne tout entière ${ }^{7} »$. Il fut un théoricien majeur de l'anarchisme et l'auteur d'une critique aussi précise qu'acerbe du marxisme de son temps. L'essentiel de cette critique est contenu dans les pages de son Appel au socialisme, un texte dans lequel Landauer démonte pas à pas les principaux fondements du marxisme, depuis le socialisme scientifique jusqu'à la dictature du prolétariat en passant par la lutte des classes et la conception matérialiste de l'histoire.

Aujourd'hui peu connu en-dehors des cercles initiés, le penseur anarchiste a connu de son vivant une notoriété certaine, notamment du fait de son importante activité de rédacteur, d'orateur et de traducteur. Si les premières occurrences de son nom dans le Vorwärts ne font qu'indiquer son travail de critique littéraire (notamment dans la présentation de numéros de revue), le ton change drastiquement lorsqu'il se rapproche du groupe des «socialistes indépendants » ou «jeunes (Jungen) » et enchaîne les attaques contre la social-démocratie. Début 1892, il commence à publier dans Der Sozialist, alors «organe des socialistes indépendants » : d'abord un extrait de son livre Le prédicateur de la mort ${ }^{8}$, puis un article anonyme intitulé «La social-démocratie moralisatrice» ${ }^{9}$. Viendront ensuite plusieurs publications directement ancrées dans les débats des Jungen, notamment sur l'œuvre d'Eugen Dühring ${ }^{10}$. Moins d'un mois plus tard, ses prises de position publiques contre la socialdémocratie sont discutées pour la première fois dans les pages du Vorwärts ${ }^{11}$. À partir de là, et en particulier suite à sa participation au congrès socialiste de Zürich en 1893, Landauer deviendra une forme d'homme de paille du Vorwärts, qui évoquera ses prises de position acharnées comme une ritournelle susceptible d'amuser les lecteurs du journal. À Zürich, s'étant attiré l'ire de l'assistance en qualifiant le discours d'August Bebel de «las et boiteux », il ne pourra reprendre la parole que parce que le président de séance souhaite éviter d'en faire un «martyre ${ }^{12}$. Par la suite, le journal social-démocrate rendra compte très régulièrement de ses interventions publiques, sans jamais manquer de le tourner en dérision et

\footnotetext{
${ }^{7}$ Services de police (Polizeipräsidium), « Der Schriftsteller Gustav Landauer 1892-1902 », rapport de 1893.

${ }^{8}$ Landauer Gustav, « Eine Rede zur Zeit der Reichstagswahlen », Der Sozialist, II, no 11, 13 mars 1892, pp. 2-3 ; Landauer Gustav, Der Todesprediger, Dresden-Leipzig, Heinrich Minden, 1893, GLAS 8, pp. 31-142 (le titre du roman est une référence à Ainsi parlait Zarathoustra de Friedrich Nietzsche).

${ }^{9}$ Landauer Gustav, « Die moralische Sozialdemokratie », Der Sozialist, II, no 34, 20 août 1892, pp. 3-4.

${ }^{10}$ Landauer Gustav, « Referat über Eugen Dühring "Kursus der National- und Sozialökonomie" », Der Sozialist, II, n ${ }^{\circ}$ 35, 27 août 1892, GLAS 2 ; « Dühringianer und Marxist », Der Sozialist, II, n 43, 22 octobre 1892, GLAS 2 .

11 «Die Mechaniker», Vorwärts, 9, n 248, 15 novembre 1892, p. 6.

12 «Aus der Schweiz », Vorwärts, 10, n 2, 3 janvier 1893, p. 5.
} 
de relever «l'esprit borné [...] qui caractérise monsieur Gustav Landauer dès qu'il en vient à parler de ses adversaires sociaux-démocrates $»^{13}$ :

Là-dessus, Landauer embraya sur ses invectives habituelles et ridicules à l'encontre de la social-démocratie. [...] En conclusion, Landauer alla encore chercher quelques insultes qu'il avait oubliées auparavant ${ }^{14}$.

Celui qui devint «l'inévitable Landauer ${ }^{15}$ » ne perdit cependant pas tout crédit auprès du Vorwärts, qui continua d'évoquer ses interventions et certaines de ses publications (quelquefois dans des encarts publicitaires, mais également dans les pages rédactionnelles). Plusieurs de ses textes furent même publiés par le Vorwärts, mais toujours sous la forme de lettres ouvertes, introduites par une formule du type «Monsieur Gustav Landauer nous écrit ». Quelquefois publiées sans commentaire ${ }^{16}$ ou avec une simple précision de la rédaction du Vorwärts ${ }^{17}$, il arrivera également que sa lettre soit insérée dans un article pour s'y voir vertement critiquée $^{18}$. Le seul texte véritablement signé du nom de l'auteur est un droit de réponse faisant suite à une série d'articles à l'intérieur desquels il est «si fréquemment apostrophé personnellement qu'[il] demande le droit de dire quelques mots $»^{19}$.

Il faut enfin noter que le Vorwärts fit parfois front commun avec Landauer, lorsque cela servait la cause du journal. Il fut ainsi soutenu à l'occasion de ses déboires avec la presse « bourgeoise $^{20}$ » et la justice ${ }^{21}$, quitte à souligner à mots couverts l'acharnement de la police ${ }^{22}$ à l'encontre des anarchistes. Le Vorwärts n'en restera pas moins un «opposant politique résolu de monsieur Landauer ${ }^{23}$ », se positionnant systématiquement «en opposition de principe au [...] Sozialist $^{24} \gg$ dont Landauer devint rédacteur en chef à partir de 1893.

\section{CONTRE LE PARTI : LANDAUER ET LES «SOCIALISTES INDÉPENDANTS »}

Gustav Landauer est parfois présenté comme un renégat de la social-démocratie, venu à l'anarchisme au terme de ses désillusions ${ }^{25}$. Pourtant, s'il est vrai que de nombreux anarchistes ont forgé leurs convictions libertaires en rupture avec le marxisme, Landauer n'a jamais été membre du parti social-démocrate : «sans mettre de nom dessus, il se trouva donc que j'étais un anarchiste avant de devenir un socialiste, et que je suis l'un des rares à ne pas

\footnotetext{
13 «Herr Gustav Landauer », Vorwärts, 13, n 298, 20 décembre 1896, p. 1.

14 «Versammlungen », Vorwärts, 13, n 247, 21 octobre 1896, p. 2.

${ }^{15}$ Id. ; voir également « Nachdem es den Anarchisten... », Vorwärts, 13, n ${ }^{\circ}$ 57, 7 mars 1896, p. 4.

16 Landauer Gustav, «Erklärung », Vorwärts, 15, n 262, 8 novembre 1898, pp. 1-2; Landauer Gustav, «Anarchistisches », Vorwärts, 16, n ${ }^{\circ} 33,8$ février 1899, p. 4 ; Landauer Gustav, « Anarchistenhetze », Vorwärts, 28, n 164, 16 juillet 1911, p. 2 ; Landauer Gustav, «Spitzelzensur? », Vorwärts, 29, n 58, 9 mars 1912, p. 3.

${ }^{17}$ Landauer Gustav, «In der Angelegenheit des ausgewiesenen Anarchisten Großmann », Vorwärts, 13, n 254, 29 octobre 1896, p. 1 .

18 «Zahme Anarchisten », Vorwärts, 12, n 33, 8 février 1895, pp. 1-2.

${ }^{19}$ Landauer Gustav, «Zu Peukerts Memoiren », Vorwärts, 31, nº 65, 7 mars 1914, p. 2.

${ }^{20}$ «Dem Ziethen-Komitee », Vorwärts, 15, n 279, 29 novembre 1898, p. 1.

21 «Wegen Aufreizung», Vorwärts, 10, n 301, 23 décembre 1893, p. 3 ; «Den Prozeß gegen Landauer», Vorwärts, 15, $\mathrm{n}^{\mathrm{o}} 281,1^{\mathrm{er}}$ décembre 1898, p. 2 ; «Zum Prozeß Landauer », Vorwärts, 16, nº 67, 19 mars 1899 , p. 2.

22 «Anarchistisches », Vorwärts, 16, nº 8, 10 janvier 1899, p. 3.

23 «Moralische Verkommenheit», Vorwärts, 11, n 167, 21 juillet 1894, p. 2.

24 «Anarchistisches », Vorwärts, 12, n 179, 3 août 1895, p. 2.

25 Voir par exemple Marshall Peter H., Demanding the impossible: a history of anarchism, London, Harper Collins, 1992, p. 410.
} 
avoir emprunté le chemin passant par la social-démocratie $»^{26}$. Revendiquant à la fois les qualificatifs d'anarchiste et de socialiste, il ne s'est jamais reconnu dans la voie socialdémocrate incarnée par le parti marxiste, et il a tout de suite adopté une posture critique vis-àvis de ce dernier. Ses premiers contacts se font en effet par le groupe dissident des Jungen autour de Bruno Wille, dont la fréquentation marque l'entrée de Landauer dans les débats politiques de son temps. Si la politique autoritaire du parti est en cause dans cette polémique, les termes du débat qui oppose la fraction des Jungen à l'orthodoxie du parti ne se résument pas à une opposition de principe entre «socialisme autoritaire » et « socialisme libertaire ». Une partie importante des Jungen tente ainsi de maintenir le lien avec la social-démocratie en opérant un rôle de critique interne sans se réclamer pourtant de l'anarchisme ${ }^{27}$. Ce n'est qu'à un stade déjà avancé du mouvement que la scission anarchiste opère, au sein des Jungen, en grande partie sous l'influence de Gustav Landauer ${ }^{28}$. Ce dernier fait donc partie du mouvement de contestation externe de la social-démocratie, la plus inconciliable également. Quoique cette opposition ressorte très explicitement des termes de la polémique, il semble utile d'en rappeler la genèse pour aller au-delà des «polémiques éreintantes » qui découlent inlassablement de l'argument selon lequel «les marxistes trouvent l'anarchisme petitbourgeois, contre-révolutionnaire et utopique, et les anarchistes trouvent le marxisme autoritaire, dogmatique et traître ${ }^{29}$. Contre cette posture de principe, il semble plus judicieux d'étudier les influences réciproques qui ont pu se manifester dans ces échanges houleux ${ }^{30}$, de façon à faire émerger dans leur contexte les points essentiels d'une discussion féconde entre deux tendances du socialisme. Cette contextualisation permettra également de comprendre que les premières critiques landaueriennes à l'encontre du marxisme aient été formulées comme une défense de l'œuvre de Karl Marx contre les marxistes - par la suite, en particulier dans l'Appel au socialisme de 1911, Karl Marx deviendra cependant le nom par lequel Landauer dénoncera certaines tendances structurelles de la social-démocratie de son époque, à commencer par son attentisme et son scientisme.

Le « rassemblement massif » qui donne au Vorwärts la première occasion de mentionner l'action de Landauer est commenté en ces termes : «Au terme de son exposé, monsieur Landauer trouva bon de s'en prendre également à la social-démocratie, affirmant qu'elle aurait cessé de se situer sur le terrain du Manifeste communiste ${ }^{31}$. Cela souligne que la première critique de Landauer à l'encontre du SPD n'est pas dirigée contre l'œuvre de Marx, mais bien «contre ses disciples et épigones, les marxistes ${ }^{32}$. Il vante à l'envi les mérites de l'œuvre du premier Marx, et considère alors « la force et la passion » comme les principales qualités de l'auteur ${ }^{33}$. Du Manifeste communiste, il retient la puissance du geste ; quant au Capital, il fait du chapitre sur l'accumulation du capital le « discours de fête » de son $1^{\mathrm{er}}$ mai derrière les barreaux ${ }^{34}$.

\footnotetext{
${ }^{26}$ Landauer Gustav, « Vor fünfundzwanzig Jahren », Der Sozialist, V, nº 12, 15 juin 1913, GLAS 2, p. 91.

27 Wienand Peter, «Revoluzzer und Revisionisten. Die "Jungen" in der Sozialdemokratie vor der Jahrhundertwende », Politische Vierteljahresschrift, 17, n 2, 1976, pp. 228-231.

28 Sur ce point, cf. Müller Dirk H., Idealismus und Revolution. Zur Opposition der Jungen gegen den sozialdemokratischen Parteivorstand 1890 bis 1894, Berlin, Colloquium Verlag, 1975, pp. 151-168.

29 Kuhn Gabriel, «Anarchismus und Marxismus revisited», sur kritisch-lesen.de, https://kritischlesen.de/rezension/anarchismus-und-marxismus-revisited, 7 juillet 2011, consulté le 30 janvier 2018, $\S 1$.

${ }^{30}$ Linse Ulrich, Organisierter Anarchismus im deutschen Kaiserreich von 1871, Berlin, Duncker \& Humblot, 1969, pp. 40-66.

31 «Die Mechaniker », art. cit.

${ }^{32}$ Landauer Gustav, « Dühringianer und Marxist », art. cit., GLAS 2, p. 117.

${ }^{33}$ Ibid., p. 116.

${ }^{34}$ Cf. la lettre de Landauer à Margarethe Leuschner du 24 avril 1894, reproduite dans Briefe und Tagebücher 1884-1900, Knüppel Christoph (dir.), Göttingen, V\&R Unipress, 2017, pp. 389-390: «Le premier mai en
} 
Qu'une remarque personnelle me soit permise. Je l'ai souvent lu [le Manifeste communiste], et il m'a toujours ébloui. Cela faisait quelques mois que j'avais laissé Marx de côté. Et hier j'ai repris en main le Manifeste communiste avec la ferme intention de ne pas m'en laisser imposer de nouveau. Je voulais le maîtriser, le critiquer. Eh bien franchement, j'ai eu le dessous. Le Manifeste communiste est et reste un trait de génie, une construction grandiose ${ }^{35}$.

Cette admiration pour le geste de Marx motive les premières critiques de Landauer à l'encontre des sociaux-démocrates, auxquels il reproche d'avoir renoncé à la révolution sociale au profit d'un simple réformisme politique. Dès lors, on pourrait croire à une dissension qui se situerait initialement au sein du mouvement marxiste - lequel traversera, quelques années plus tard, sa grande «querelle du réformisme (Reformismusstreit) ${ }^{36}$. Pourtant, la suite de son commentaire sur le Manifeste communiste met en lumière le désaccord fondamental qui sépare la voie landauerienne de la voie marxienne :

«À la place de l'ancienne société bourgeoise, avec ses classes et ses antagonismes de classe, surgit une association où le libre développement de chacun est la condition du libre développement pour tous. »Cet objectif est celui de tous les anarchistes et socialistes libres, et il n'a que rarement été formulé d'une manière plus concise ni plus belle. Mais qu'en est-il du chemin ? Le chemin vers cette absence de domination est, chez Marx, la domination, le chemin vers cette absence d'État est chez Marx l'État ${ }^{37}$.

Sur le rapport à l'État et à la domination, Landauer assimile donc la position de Marx et celle de ses disciples pour s'en faire l'opposant - posant ainsi les bases d'une distinction fondamentale entre socialisme libertaire et social-démocratie. C'est à l'occasion des congrès socialistes de Zürich en 1893 et de Londres en 1896 que le schisme sera consommé au sein du socialisme entre une voie anarchiste et celle, dominante, prônée par la social-démocratie. Le scandale qui entoura l'exclusion des délégués anarchistes lors de ces rassemblements internationaux est relaté dans les organes de presse des différents protagonistes. Le premier épisode de cette controverse coïncide aussi avec une scission au sein du mouvement des Jungen entre une voie se revendiquant de l'anarchisme et du socialisme, et une autre refusant la première épithète. Cette querelle de mots sera reprise et alimentée par le Vorwärts : si la voix anonyme du journal social-démocrate évoque avec une prudente réserve « les anarchistes qui se désignent aujourd'hui comme socialistes anarchistes ${ }^{38}$, le président du parti August Bebel martèle quant à lui l'incompatibilité des deux directions. Pour preuve, ce compte-rendu d'une discussion au cours de laquelle Bebel et Landauer étaient successivement intervenus :

Mais cela m'a semblé être le sommet de la contradiction lorsqu'il [Landauer] a affirmé être anarchiste et socialiste selon l'humeur. Qui considère le monde

\footnotetext{
prison ! [...] Je déposai alors, à l'endroit où j'avais coutume de m'asseoir, le livre premier de Marx et j'ouvris le chapitre sur l'accumulation du capital. Ce fut le discours de fête. »

${ }^{35}$ Landauer Gustav, «Manchesterfreiheit - Staatshilfe - Anarchie. Politische Unfreiheit - Politische Mitarbeit Negation des Staats », Der Sozialist, III, n 26, 24 juin 1893, GLAS 2, p. 135.

${ }^{36}$ Cet épisode de crise interne à la social-démocratie est décrit dans l'Histoire générale du socialisme de Jacques Droz, Paris, Presses universitaires de France, 1979, vol. 2/4, pp. 37-51 et dans l'ouvrage de Winock Michel, Le socialisme en France et en Europe, XIX ${ }^{e}-X X^{e}$ siècle, Paris, Seuil, 1992, pp. 111-115.

${ }^{37}$ Landauer Gustav, « Manchesterfreiheit - Staatshilfe - Anarchie. Politische Unfreiheit - Politische Mitarbeit Negation des Staats », art. cit., GLAS 2, p. 135. Le passage cité apparaît à la fin de la seconde partie du Manifeste du Parti communiste (1848), Paris, Union générale d'éditions, 1962, p. 47.

38 «Versammlungen », art. cit.
} 
ainsi, en fonction de l'humeur du moment, avec celui-là je n'ai pas à discuter plus avant (applaudissements nourris). ${ }^{39}$

S'il joue sur la veine comique pour ridiculiser son adversaire, Bebel sait bien que la voie des « anarchistes-socialistes » ne consiste pas à alterner de l'une à l'autre position, mais bien à faire cohabiter les deux. Cela fit l'objet d'un débat interne au Sozialist dans lequel Landauer intervint la même année ${ }^{40}$. Suite aux deux congrès dont il fut exclu, Landauer rappelle que «nous, les anarchistes, en Allemagne, nous nous considérons tous comme des socialistes ${ }^{41}$. Cette affirmation fut la ligne directrice de sa protestation contre l'exclusion des mandataires anarchistes. Selon Landauer, les seuls à pouvoir douter que les anarchistes soient des socialistes étaient la police, la bourgeoisie et « les lecteurs fourvoyés du Vorwärts ainsi que de l'ensemble des journaux sociaux-démocrates ${ }^{42}$. Ce qu'avait d'inconcevable cet argument pour le référentiel marxiste ressort assez de cet article du Vorwärts, qui rapporte l'intervention de Landauer en ces termes : «Les anarchistes devraient être admis si les anarchistes, comme lui, se trouvaient être des socialistes (rire général) ${ }^{43}$. Landauer, qui déplore que «dans chacun de ses numéros, le Vorwärts récuse toute, mais vraiment toute affinité entre le socialisme et l'anarchisme ${ }^{44}$, fera l'objet d'une série de moqueries similaires de la part des rédacteurs du Vorwärts. Lorsque le sous-titre du journal Der Sozialist devient en 1895 « organe pour l'anarchisme-socialisme " ${ }^{45}$, ils relèvent ainsi : «La devise du Sozialist (au fait, pourquoi pas Anarchist ?) devrait être : "Deux âmes, hélas ! se partagent mon sein" »" Par cette référence au Faust de Goethe, le Vorwärts cherche encore à faire passer pour intenable la posture anarchiste-socialiste, là où Landauer revendique la synonymie des deux notions, d'accord en cela avec la plupart des anarchistes :

Anarchie, en effet, est, avant tout, synonyme de socialisme. L'anarchiste est, en premier lieu, un socialiste qui vise à abolir l'exploitation de l'homme par l'homme. L'anarchisme n'est pas autre chose qu'une des branches de la pensée socialiste. Une branche où prédominent le souci de la liberté, la hâte d'abolir l'État. ${ }^{47}$

Si Landauer n'affirme pas autre chose, ses articles portant sur la question «Comment nous nommons-nous ? ${ }^{48}$ marqueront «l'incompatibilité pratique des deux mouvements ${ }^{49}$ au sein de la rédaction du Sozialist. Ceux qui affirmaient encore au début des années 1890 que

\footnotetext{
39 «Attentate und Sozialdemokratie », Vorwärts, 15, n ${ }^{\circ} 259,4$ novembre 1898, p. 3.

${ }^{40}$ Landauer Gustav, «Wie nennen wir uns? », Der Sozialist, III, n ${ }^{\circ} 14,1^{\text {er }}$ avril 1893, GLAS 2, pp. 124-127; Landauer Gustav, «Zur Frage: Wie nennen wir uns? », Der Sozialist, III, n 16, 15 avril 1893, GLAS 2, pp. 127 131.

${ }^{41}$ Landauer Gustav, «Von Zürich bis London. Bericht über die deutsche Arbeiterbewegung an den Londoner Internationalen Kongress », Der Sozialist, VI, n 29, 18 juillet 1896, GLAS 2, p. 215, traduit la même année par Doris Wess-Zhook sous le titre « De Zurich à Londres. Rapport sur le mouvement ouvrier allemand au Congrès international de Londres », réédité in Gomez Freddy (dir.), Gustav Landauer, un anarchiste de l'envers, Paris, Éditions de l'éclat / À contretemps, 2018, p. 153.

${ }^{42}$ Landauer Gustav, «Zur Frage: Wie nennen wir uns? », art. cit., GLAS 2, p. 128.

43 «Internationaler Arbeiter-Kongreß in Zürich », Vorwärts, 10, n 185, 9 août 1893, p. 1.

${ }^{44}$ Landauer Gustav, « Genug, ihr Heuchler! », Der Sozialist, VIII, n 39, 24 septembre 1898, GLAS 2, p. 236.

${ }^{45}$ Le journal Der Sozialist a eu les sous-titres suivants : «Organe des socialistes indépendants » (1891-1893),

«Organe de tous les révolutionnaires »(1893-1895), «Organe des anarchistes allemands » (1895), «Organe pour l'anarchisme-socialisme » (1895-1899), « Mensuel anarchiste » (1899). De 1909 à 1915, Landauer relance sa publication avec le sous-titre « Organe de l'Alliance socialiste ».

46 « Anarchistisches », Vorwärts, 12, n 181, 6 août 1895, p. 2. La citation est tirée de Faust (1808), traduit par Gérard de Nerval, Paris, Garnier frères, 1877, p. 59.

${ }^{47}$ Guérin Daniel, L'anarchisme : de la doctrine à la pratique (1965), Paris, Gallimard, 1981, p. 15.

${ }^{48}$ Landauer Gustav, «Wie nennen wir uns?», art. cit. ; et «Zur Frage: Wie nennen wir uns? », art. cit.

${ }^{49}$ Müller Dirk H., Idealismus und Revolution, op. cit., p. 163.
} 
«nous n'irons pratiquement pas plus loin avec le principe anarchiste-individualiste ${ }^{50}$, tout en insistant sur «le caractère inconciliable des différences organisationnelles entre les indépendants sociaux-démocrates et les anarchistes $»^{51}$, quittent alors la rédaction. Ils laissent ainsi le champ libre à l'anarchisme-socialisme. Cette controverse joua un rôle certain dans l'histoire du mouvement socialiste, puisqu'un an après la parution des articles de Landauer consacrés à la question des dénominations, le Vorwärts charge le philosophe russe Gueorgui Plekhanov de rédiger une brochure intitulée Anarchisme et socialisme, traduite en de nombreuses langues ${ }^{52}$. La dispute laissera également des traces dans l'œuvre de Landauer qui, sans jamais renier l'un ou l'autre des deux qualificatifs, et présentant « anarchisme comme le mot qui convient le mieux pour désigner [sa] conception de la vie » dans sa correspondance privée $^{53}$, se montrera par la suite très prudent dans l'usage de ces notions. Il écrit ainsi que « l'anarchie n'est qu'un autre nom pour le socialisme, moins bon du fait de sa négativité et de son équivocité particulièrement forte ${ }^{54}$. C'est probablement pour cette raison, mais également du fait d'un glissement progressif d'une posture contestataire vers une posture de proposition, que la seconde moitié de l'œuvre de Landauer laisse une place plus significative à la notion de socialisme qu'à celle d'anarchisme.

\section{L’«INSIGNIFIANCE » POLITIQUE D’UNE FIGURE LITTÉRAIRE}

La manière dont Landauer est dépeint dans le Vorwärts vise le plus souvent à minimiser le poids politique de l'auteur, notamment lorsqu'il est présenté comme figure littéraire. Il faut dire que, s'il peut rétrospectivement être considéré comme un philosophe et un activiste, le jeune Landauer ne présentait pas encore ce visage : au moment où son œuvre de philosophe ne repose sur aucune publication majeure, et où son activisme politique embrasse déjà la direction du «socialisme culturel ${ }^{55}$ qui le caractérisera, Landauer signe certaines de ses lettres «Gustav Landauer, écrivain ${ }^{56}$. Il est donc cohérent qu'il soit alors présenté comme tel. Lorsqu'il prend la direction du supplément littéraire au Sozialist, il se voit taxer de « l'aide de camp littéraire de monsieur Wiese ${ }^{57}$. De même, le seul semblant de compliment qui lui est adressé de son vivant est une critique larvée de ses aspirations politiques : «le très talentueux rédacteur Landauer ${ }^{58}$ est présenté comme un simple homme de lettres ${ }^{59}$ dont les prétentions à changer la société prêtent à rire.

L'inévitable monsieur Landauer ! Ce redoutable révolutionnaire s'est donc déjà mué en le fondateur d'un groupe de lecture, et au vu du roman dont il était déjà

\footnotetext{
${ }^{50}$ Der Sozialist, I, n ${ }^{\circ} 5,13$ décembre 1891.

${ }^{51}$ Müller Dirk H., Idealismus und Revolution, op. cit., p. 138.

${ }^{52}$ Plekhanov Georges, Anarchisme et socialisme, Paris, Librairie de l'Humanité, 1923 (parution initiale à Berlin, Verlag der Expedition des « Vorwärts », 1894).

${ }^{53}$ Landauer Gustav, lettre à Paul Eltzbacher, 2 avril 1900, GLAS 2, p. 273.

${ }^{54}$ Landauer Gustav, «Les douze articles de l'Alliance socialiste » (1912), Angaut Jean-Christophe et Lucet Anatole (trad.), in Appel au socialisme, op. cit., p. 203.

${ }^{55}$ Kalz Wolf, Gustav Landauer: Kultursozialist und Anarchist, Meisenheim am Glan, Hain, 1967.

56 Notamment dans le Vorwärts : cf. Landauer Gustav, «Erklärung », art. cit., p. 2. L'activité littéraire de Landauer est exposée dans l'article de Despoix Philippe, «De la scène à l'histoire : l'antipolitique de Gustav Landauer », Romantisme, 25, n 87, 1995, pp. 13-28. Une analyse détaillée en est proposée dans l'ouvrage de Kaiser Corinna, Gustav Landauer als Schriftsteller: Sprache, Schweigen, Musik, Berlin, De Gruyter, 2014.

57 «Zahme Anarchisten », art. cit., p. 1.

58 «Gerichts-Zeitung », Vorwärts, 11, n 295, 19 décembre 1894, p. 2.

${ }^{59} \mathrm{Si}$ Landauer pense un temps devoir se détourner de l'art pour se consacrer à la politique et au changement social, il effectuera finalement la synthèse de ces deux directions dans sa conception d'un socialisme culturel. Sur ce point, cf. Fähnders Walter, "Gustav Landauer - Anarchisme, littérature, révolution », in Pessin Alain et Terrone Patrice (dir.), Littérature et anarchisme, Toulouse, Presses universitaires du Mirail, 1998, pp. 365-386.
} 
l'auteur, la tragi-comédie semble encore loin de toucher à sa fin. Le Braunschweiger Volksfreund a récemment esquissé une description de ce roman chaleureusement intitulé : Le prédicateur de la mort ; en tant qu'amis du bon humour, nous ne pouvions la passer sous silence $[\ldots] .{ }^{60}$

Cette manière de présenter l'auteur comme un artiste inconstant contribue à faire douter de son impact politique. Un mécanisme similaire opère encore au sujet du rôle tenu par les poètes au cours de la révolution allemande, en particulier lors de la république des conseils de Bavière, ceux-ci étant souvent présentés comme de doux rêveurs ${ }^{61}$. Mais dans le cas de Landauer, cette campagne de délégitimation s'est doublée d'une autre accusation : celle de porter le titre d' «expert en confusion (Konfusionsrath) » ${ }^{62}$ s'adonnant à des « arguties talmudiques» et des «explication jésuitiques complémentaires sur le concept d'action politique ${ }^{63}$. Cette caractérisation de la parole de Landauer n'aura jamais quitté celui qui, au cœur de la révolution allemande, aurait fait publier «de grands dessins cubistes, d'interminables articles sur des colonnes entières concernant le système des conseils et autres mécanismes théoriques ${ }^{64}$, totalement hermétiques aux ouvriers ${ }^{65}$. Pourtant, ce que ses adversaires interprétèrent comme une insuffisance ou un manque de clarté traduisait en réalité une différence de stratégie discursive profonde. L'auteur anarchiste s'est ainsi toujours inscrit en faux contre les « professeurs » qui prétendaient énoncer la vérité sur la société ou formuler des lois de l'histoire, préférant à ceux-là les «prophètes » dont le message, suggestif et non démonstratif, ferait surgir en chacun l'aspiration à reconstruire les liens de communauté. C'est à ce titre qu'il critique «la mécanisation et la transformation de réalités fluides vivantes en lois et en concepts morts et rigides » caractéristiques du socialisme scientifique ${ }^{66}$ et qu'il revendique l'approche poétique contre la tentative de créer une science de la société :

nous sommes... mais auparavant, pauvres marxistes, prenez une chaise, asseyez-vous et tenez-vous bien ; car arrive l'effroyable ; l'effronterie arrive et en même temps, quelque chose va vous être enlevé que vous auriez brûlé de m'objecter d'un ton méprisant... nous sommes des poètes; et les charlatans de la science, les marxistes, les froids, les vides, les sans-esprit, nous voulons nous en débarrasser pour que le regard poétique, la figuration concentrée d'une manière artistique, l'enthousiasme et la prophétie trouvent place là où dorénavant ils ont à faire, à créer, à construire : dans la vie, avec les corps des êtres humains, en faveur de la vie partagée, du travail et de l'intimité des groupes, des communes, des peuples ${ }^{67}$.

L'écart entre cette façon d'envisager le changement social et la doxa marxiste de son temps était tel que l'image de l'homme de lettres ne quittera jamais Landauer dans les écrits

\footnotetext{
60 «Nachdem es den Anarchisten... », art. cit.

${ }^{61}$ Weidermann Volker, Träumer - Als die Dichter die Macht übernahmen, Köln, Kiepenheuer \& Witsch, 2017.

62 «Herr Landauer», Vorwärts, 15, n 121, 26 mai 1898, p. 2 ; «Der Kampf um die Münchener Kunstakademie », Vorwärts, 36, n ${ }^{\circ}$ 205, 23 avril 1919, p. 2. C'est un reproche qui sera également adressé au Sozialist sous la direction de Landauer. Cf. Leder Tilman, Die Politik eines «Antipolitikers »: Eine politische Biographie Gustav Landauers, Lich/Hessen, Verlag Edition AV, 2014, vol. 1/2, pp. 229-231.

${ }^{63}$ «Internationaler Arbeiterkongreß in Zürich », Vorwärts, 10, n 188, 12 août 1893, p. 2.

${ }^{64}$ Grossmann Stefan, «Gustav Landauer », Das Tagebuch, 10, n 18, 4 mai 1929, p. 736. Cf. notamment la Une des Münchner Neueste Nachrichten en date du 8 avril 1919, reproduite dans Leder Tilman, Die Politik eines «Antipolitikers », vol. 2/2, op. cit., p. 825.

65 «Der Kampf um die Münchener Kunstakademie », art. cit.

${ }^{66}$ Landauer Gustav, «Referat über Eugen Dühring "Kursus der National- und Sozialökonomie" », art. cit., GLAS 2, p. 108.

${ }^{67}$ Landauer Gustav, Appel au socialisme, op. cit., pp. 63-64.
} 
du Vorwärts : que ce soit pour moquer ou pour vanter son travail, le point de vue sera toujours exclusivement littéraire.

Ainsi, lorsqu'après sa mort, le Vorwärts mentionne le nom de Landauer ${ }^{68}$, c'est pour évoquer la réédition d'un recueil de nouvelles et d'un roman, sans pour autant mentionner ses deux écrits théoriques les plus importants, La révolution et Appel au socialisme ${ }^{69}$. Quelques années plus tard, un journaliste désigne comme relevant de son «mérite immortel» le fait d'avoir traduit L'Entr'aide de Pierre Kropotkine ${ }^{70}$. Comment expliquer que l'ouvrage considéré par l'auteur lui-même comme «le meilleur de ses écrits " ${ }^{71}$, son Appel au socialisme, n'ait été mentionné pour la première fois qu'à l'occasion des dix ans de sa mort, en passant et au même niveau que sa collection de Lettres de la Révolution française ${ }^{72}$ ? Ce texte contient pourtant sa critique la plus virulente mais aussi la plus précise du marxisme. Différentes hypothèses pourraient être avancées sur les raisons de ce silence ${ }^{73}$. Notons simplement que l'absence de références à cet ouvrage ou à son écrit de philosophie de l'histoire $L a$ révolution a contribué à occulter la théorie politique qui sous-tendait toute l'œuvre de ce penseur de la société. Du vivant de l'anarchiste, le Vorwärts soulignait déjà «l'insignifiance du "groupe Landauer" " ${ }^{74}$ sur un plan étroitement politique, c'est-à-dire du point de vue de ses effectifs. Après sa mort, on rappela que «c'était un homme qui provenait complètement du domaine de l'esprit et n'était absolument pas né pour être un dirigeant politique ${ }^{75}$. La conception politique sous-jacente à cette remarque est totalement étrangère à la conception de l'anarchiste, selon laquelle le changement social ne doit en aucun cas reposer sur des dirigeants politiques, mais bien sur l'action de personnes impliquées, unies par un esprit commun. Et lorsque finalement, près d'un quart de siècle après la mise au ban de l'anarchisme par August Bebel, la conception landauerienne du socialisme est admise parmi les différentes nuances du mouvement, c'est encore en insistant sur l'homme Landauer bien plus que sur sa conception du changement social :

\footnotetext{
${ }^{68}$ Dans les jours qui suivent l'assassinat de Landauer, le Vorwärts commence par affirmer qu'il aurait été lynché par la foule («Die Tragödie von München», Vorwärts, 36, n 226, 4 mai 1919, p. 1 ; « Die Ermordung der Geißeln bestätigt », Vorwärts, 36, n 228, 5 mai 1919, p. 2). Il faut près d'un an pour que le journal attribue les faits à une personne («Landauers Mörder freigesprochen », Vorwärts, 37, nº 166, 30 mars 1920, p. 3), et sept pour que les corps francs soient mis en cause par l'évocation de « Gustav Landauer, assassiné par des adeptes de la croix gammée» (Hyar Hans, «Die Machtfülle des Kriminalbeamten. Betrachtungen zur Magdeburger Mordaffäre », Vorwärts, 43, n 346, 25 juillet 1926, p. 3). Le fait que ces troupes aient été commanditées par Johannes Hoffmann et Gustav Noske (SPD) n'est pas mentionné. Sur cet épisode, cf. le témoignage d'un contemporain de Landauer qui prit part à la révolution en Bavière à ses côtés : Toller Ernst, Une jeunesse en Allemagne (1933), Gallissaires Pierre (trad.), Lausanne, L'Âge d'Homme, 1974, chap. XI-XVI.

${ }^{69}$ Hochdorf Max, «Denken und Dichten Gustav Landauers », Vorwärts, 40, no 86, 21 février 1923, p. 2.

${ }^{70}$ Wächter W., «Gedanken zum Ameisenkrieg in Weißensee », Vorwärts, 44, n 70, 11 février 1927, p. 1. Landauer et Kropotkine avaient été voisins à Bromley en 1901. Plusieurs «traductions autorisées » paraissent les années suivantes, dont Gegenseitige Hilfe in der Entwickelung, Landauer Gustav (trad.), Leipzig, Theodor Thomas Verlag, 1904. Pour la traduction française, dont le titre avait été suggéré par Élisée Reclus, cf. L'Entr'aide : un facteur de l'évolution, Bréal Louise (trad.), Paris, Hachette, 1906.

${ }^{71}$ Landauer Gustav, lettre à Leo Kestenberg du 16 octobre 1918, reproduite dans Gustav Landauer: Sein Lebensgang in Briefen, Buber Martin et Britschgi-Schimmer Ina (éd.), Frankfurt am Main, Rütten \& Loening, 1929, vol. 2/2, p. 278.

${ }^{72}$ Hartmann Hans, «Zum 10. Todestage Gustav Landauers », Vorwärts, 46, n ${ }^{\mathrm{o}} 202,1^{\mathrm{er}}$ mai 1929, p. 1.

${ }^{73}$ Les articles du Vorwärts n'étant généralement pas signés, une étude stylistique serait nécessaire pour identifier les différents auteurs qui assurèrent la couverture de l'anarchisme sur les différentes périodes envisagées. Plus qu'à une improbable conspiration du silence, on pourrait ainsi attribuer cette étonnante absence de Landauer à une simple évolution dans l'équipe rédactionnelle. Par ailleurs, le fait que Landauer adopte une posture moins oppositionnelle que dans les années 1890 dans ses interventions publiques a dû contribuer à n'en faire qu'un adversaire de second plan.

${ }_{74}$ «Die parteigenössischen Vertrauensleute Berlins », Vorwärts, 15, nº 119, 24 mai 1898, p. 1.

${ }^{75}$ Hartmann Hans, «Zum 10. Todestage Gustav Landauers », art. cit.
} 
Qui connaît l'œuvre de Gustav Landauer sait que ses conceptions du socialisme et de la lutte de la classe ouvrière s'écartent à bien des égards de notre position, et que ce n'était pas son genre de s'insérer et de s'intégrer dans un mouvement de masse. Pourtant, tous ceux qui ne sont pas obtus reconnaîtront que son Appel au socialisme et maints autres écrits peuvent nous apporter beaucoup. On ne voudrait pas se passer de cette tonalité dans la grande symphonie du socialisme, et encore moins voudrait-on se priver de l'homme Landauer, qui nous est documenté d'une manière si merveilleuse dans ses lettres ${ }^{76}$.

Dans le même numéro, l'article qui reconnaît que «pour nous, socialistes, Landauer signifie encore une question particulière et un aiguillon particulier » explique néanmoins son échec par le fait qu'il n'aurait pas vu « l'essentiel du message de Karl Marx » ${ }^{77}$. Et la semaine suivante, dans le compte-rendu d'une réunion organisée à la mémoire de Landauer, le Vorwärts déplore un "défaut de la cérémonie », qui aurait laissé de côté « le linguiste, [...] le critique littéraire » au profit du seul aspect que le journal marxiste n'aura, sur toutes ces années, quant à lui jamais développé : « la voix seule est donnée au révolutionnaire politique et culturel $»^{78}$.

\section{CONTRE LA LUTTE DES CLASSES : UN « POINT DE VUE PUREMENT BOURGEOIS »?}

Contrairement à ce que l'on aurait pu attendre, le travail de délégitimation de Gustav Landauer s'est rarement appuyé sur sa classe sociale d'origine - cet argument se serait facilement retourné contre une partie des meneurs marxistes de l'époque. Né dans une famille de commerçants et formé dans les universités de Strasbourg, Heidelberg et Berlin, Landauer est de fait issu de la moyenne bourgeoisie intellectuelle de son temps ${ }^{79}$. Cet argument n'est pourtant relevé qu'une fois dans un compte-rendu relatif aux premières interventions publiques de Landauer :

La manière dont l'anarchiste Landauer fut expédié par un intervenant est digne d'intérêt. Celui-ci déclara [...] que Landauer était le représentant d'un point de vue purement bourgeois, qu'il n'avait jamais travaillé dans une usine ni fait l'expérience, en tant que travailleur, de ce qu'il en était de la libre individualité du travailleur. Qu'il fallait que le travailleur acquière une telle individualité dans la lutte des classes. ${ }^{80}$

Landauer répondra explicitement à ces accusations, non en cherchant à faire amende honorable par le reniement de ses origines, mais en soulignant les défauts de la grille de lecture classiste ${ }^{81}$. En refusant de se donner pour tâche «la politique prolétarienne [et] la lutte des classes ", qu'il considère comme des agents de maintien de l'ordre existant, des

\footnotetext{
${ }^{76}$ Fabian Walter, «Gustav Landauers Briefe », Vorwärts, 46, $\mathrm{n}^{\mathrm{o}} 202,1^{\mathrm{er}}$ mai 1929, p. 1. Il est ici question des deux volumes de lettres publiés par Martin Buber : Gustav Landauer: Sein Lebensgang in Briefen, op. cit.

${ }^{77}$ Hartmann Hans, «Zum 10. Todestage Gustav Landauers », art. cit.

${ }_{78}^{78}$ F. S., «Zum Gedächtnis Landauers. Matinee in der Volksbühne », Vorwärts, 46, n 209, 6 mai 1929, p. 3.

${ }^{79}$ S'il arrive quelques années plus tard qu'on lui attribue (par ironie ou par méconnaissance) le titre de «docteur»(«Nachdem es den Anarchisten... », art. cit.), il est qualifié dans une recension sur l'œuvre de Peukert dont il avait rédigé la préface de «noble anarchiste de sensibilité éthique » (Fischer Richard, « $\mathrm{Zu}$ Peukerts Memoiren », Vorwärts, 31, n 65, 7 mars 1914, p. 3).

80 «In der vorgestrigen Versammlung », Vorwärts, 10, n 174, 27 juillet 1893, p. 2.

${ }^{81}$ Landauer Gustav, «Die geschmähte Philosophie », Der Sozialist, III, n 29, 15 juillet 1893, GLAS 5, pp. 123124.
} 
«adjuvants nécessaires du capitalisme et de la puissance étatique ${ }^{82}$, Landauer cherche à revaloriser certaines classes intermédiaires comme celle des paysans. Alors qu'ils sont qualifiés de «petits paysans arriérés » par le Vorwärts, Landauer insiste pour qu'ils soient considérés comme «travailleurs et [...] pauvres et [...] exploités ${ }^{83}$. À l'inverse, Landauer met en doute la capacité de la classe ouvrière à s'extraire du capitalisme par les moyens que lui offre la social-démocratie. C'est dans ce sens qu'il écrit que « les ouvriers ne sont pas une classe révolutionnaire, mais un amas de pauvres hères, voués à vivre et mourir au sein du capitalisme $»^{84}$. Ce n'est pas le mépris de classe qui parle $\mathrm{ici}^{85}$, mais une critique que l'on retrouvera au sein du marxisme à propos des syndicats et des organisations ouvrières :

Les luttes de classes sont avant tout des luttes au sein du capitalisme: le prolétariat lutte pour ses conditions d'existence en tant que prolétariat, il s'agit de l'augmentation des salaires ou de l'amélioration des conditions de travail. Partant, les luttes des classes ne sont pas l'indice d'une déficience particulière du capital ou d'une révolution prochaine, mais bien la forme normale de l'opposition entre bourgeoisie et prolétariat. ${ }^{86}$

\section{«GUSTAV LANDAUER, LE RÉVOLUTIONNAIRE PAR EXCELLENCE »}

Hérétique parmi les socialistes comme parmi les anarchistes, «le fameux Landauer » fut remarqué dans les cercles sociaux-démocrates de son temps, mais toujours sa voix y fut minimisée, son propos relégué à une subversion sans profondeur :

La présence ici de ces gens est inouïe et scandaleuse, Landauer a calomnié comme tous ils calomnient, car s'ils ne calomniaient pas, ils n'existeraient $\operatorname{pas}^{87}$.

Lors du congrès du SPD en 1896 à Gotha, les anarchistes sont présentés par Bebel comme « une poignée de trublions ${ }^{88}$ ", à l'image de Landauer qui avait été désigné comme le «perturbateur de congrès venu de Berlin » quelques jours auparavant ${ }^{89}$. Si cette attaque vise avant tout à décrédibiliser les anarchistes, elle est également révélatrice d'une différence radicale entre les deux directions du socialisme sur la question du rapport à l'action. En effet, les «agitateurs » anarchistes n'ont eu de cesse d'accuser le parti social-démocrate et sa doctrine de se complaire dans l'attente. De fait, le matérialisme historique d'alors défend l'existence d'un mécanisme devant conduire, par l'approfondissement de la voie capitaliste, à un nécessaire renversement des conditions. C'est ce qu'exprimait déjà Engels dans son AntiDühring : «Le rapport capitaliste n'est pas supprimé, il est au contraire poussé à son comble.

\footnotetext{
${ }^{82}$ Landauer Gustav, «Les douze articles de l'Alliance socialiste » (1908), Angaut Jean-Christophe et Lucet Anatole (trad.), in Appel au socialisme, op. cit., p. 199.

${ }^{83}$ Landauer Gustav, «Der Aufruhr in der Champagne », Der Sozialist, III, n 10, 15 mai 1911, GLAS 1, p. 91. Le terme allemand «Arbeiter » désigne tant l'ouvrier que le travailleur au sens large. En proposant d'inclure les paysans dans la classe des «travailleurs », Landauer dénonce le fait que le socialisme marxiste se concentre exclusivement sur les ouvriers du prolétariat industriel, au détriment d'autres formes de travail.

${ }^{84}$ Landauer Gustav, Appel au socialisme, op. cit., p. 106.

${ }^{85}$ Landauer s'en défendra dans l'article «Individualismus », art. cit., GLAS 2, p. 86.

${ }^{86}$ Heinrich Michael, Kritik der politischen Ökonomie: eine Einführung, $3^{\mathrm{e}}$ éd., Stuttgart, Schmetterling Verlag, 2010, p. 197 ; cité dans Kellermann Philippe, «Vom Geist und geistlosen Zuständen. Ein Versuch über den Anarchisten Gustav Landauer », Grundrisse. Zeitschrift für linke Theorie \& Debatte, n 17, 2006, pp. 5-12.

87 « Aus der Schweiz », art. cit., p. 6.

${ }^{88}$ « Parteitag der sozialdemokratischen Partei Deutschlands. Dritter Verhandlungstag », Vorwärts, 13, $\mathrm{n}^{\mathrm{o}} 242,15$ octobre 1896, p. 3.

${ }^{89}$ «Aus Stuttgart», Vorwärts, 13, n 206, 3 septembre 1896, p. 3.
} 
Mais, arrivé à ce comble, il se renverse. ${ }^{90}$ Les épigones marxistes de la Deuxième internationale ont majoritairement souscrit à cette thèse et se sont pour certains réfugiés dans une « résignation maladive », allant jusqu'à renoncer dans leur combat à la simple notion de « revendication $»^{91}$.

Les sociaux-démocrates en Allemagne ont bien souvent donné la preuve, dans ces trois dernières années, qu'ils ont cessé de réveiller l'esprit révolutionnaire qui sommeille dans les masses et de l'élever à une claire conscience ${ }^{92}$.

Cette dernière accusation vise particulièrement Karl Kautsky ${ }^{93}$, le théoricien de l'orthodoxie marxiste dans le cadre de la Deuxième internationale, qui écrivait alors : «Nous ne songeons $[. .$.$] nullement à provoquer ou à préparer une révolution { }^{94}$. » Au lendemain de la révolution allemande, il réaffirmait «la stérilité de toute tentative d'introduire cette dernière forme de production [socialiste] à la place de la précédente [capitaliste] tant que les conditions ne sont pas assez mûres pour le faire ${ }^{95} »$. Au vu de cette posture résolument attentiste, on comprend ce qu'avait de péjoratif le qualificatif choisi par August Bebel pour désigner «Gustav Landauer, le révolutionnaire par excellence ${ }^{96}$ ». Contre cette résignation face aux soi-disant lois de l'histoire, Landauer prônait une révolution effective, ici et maintenant. Bien loin des théories du grand soir $^{97}$, son socialisme reposait sur les petits commencements, sur la volonté des individus et sur l'expérimentation à petite échelle.

\section{« DOCILES ANARCHISTES »? UNE EFFICIENCE SOCIALE ANTIPOLITIQUE DANS L'EMPIRE ALLEMAND}

Cette valorisation d'un socialisme ne passant pas exclusivement par les institutions politiques a conduit Landauer à participer à la constitution de la première coopérative de consommateurs-producteurs de Berlin ${ }^{98}$. Il prit ainsi part à ce que la social-démocratie avait déjà disqualifié comme «une utopie petit-bourgeoise ancestrale ${ }^{99}$ »: la voie des «petites coopératives de production inoffensives ${ }^{100} »$. Pourtant, contrairement à ce que lui opposait la doxa marxiste, cet investissement par Landauer du projet des coopératives ne signifiait en rien son renoncement à un changement profond et radical de la société. Bien au contraire, c'était

\footnotetext{
${ }^{90}$ Ce texte de 1878 sera repris dans une autre publication, initialement destinée à un lectorat français : Engels Friedrich, Socialisme utopique et socialisme scientifique. Le développement du socialisme de l'utopie à la science (1880), Paris, Éditions sociales, 1977, p. 173.

${ }^{91}$ Landauer Gustav, « Dühringianer und Marxist », art. cit., GLAS 2, p. 118.

${ }^{92}$ Landauer Gustav, « De Zurich à Londres », art. cit., p. 148 (traduction modifiée).

93 «Comme quantité d'autres anti-autoritaires de son époque, [Landauer] assimile le marxisme à ce qu'en fit Karl Kautsky, le grand "vulgarisateur" du socialisme scientifique, au tournant du siècle. » (Pinkus Paul Theodor, «Gustav Landauer», in Briefe nach der Schweiz: Gustav Landauer, Erich Mühsam, Max Höelz, Peter Kropotkin, Zürich, Limmat-Verlag, 1972, p. 12).

${ }^{94}$ Kautsky Karl, «Ein sozialdemokratischer Katechismus », Die neue Zeit, n 12, décembre 1893, p. 368, cité et traduit dans Kautsky Karl, Le chemin du pouvoir (1909), Fay Victor (trad.), Paris, Éditions Anthropos, 1969, p. 67.

${ }_{95}$ Kautsky Karl, Terrorisme et communisme : contribution à l'histoire des révolutions (1919), Stchoupak Nadine (trad.), Paris, Éditions du Sandre, 2007, p. 155. Contre cette posture, Landauer écrit : «Je considère que cela est absolument faux, car je suis de l'avis hérétique que les conditions économiques pour le socialisme n'ont jamais fait défaut » («Morgen oder übermorgen? », Der Sozialist, III, n 40, 30 septembre 1893, GLAS 2, p. 42).

${ }^{96}$ Bebel August, «Erklärung », Vorwärts, 10, n ${ }^{\circ} 232,3$ octobre 1893, p. 3.

97 Sur ce point, on pourra lire l'article de Löwy Michael, «Gustav Landauer, révolutionnaire romantique », Tumultes, 1, $\mathrm{n}^{\mathrm{O}}$ 20, 2003, pp. 93-103.

${ }^{98}$ Landauer Gustav, Ein Weg zur Befreiung der Arbeiter-Klasse, Berlin, Adolf Marreck, 1895, GLAS 14.

99 «Zahme Anarchisten », art. cit., p. 1.

100 «Anarchistisches », art. cit.
} 
pour lui la réalisation de ce qu'il n'eut de cesse d'affirmer, à savoir que «ce qui mènera effectivement au but doit [...] commencer petit ${ }^{101}$. Cette idée fut au cœur de l'Alliance socialiste qu'il cofonda en 1908, et qui considérait que « la forme fondamentale de la culture socialiste est l'alliance des communes économiques qui, d'une manière autonome, produisent et échangent entre elles dans la justice ${ }^{102}$. Loin de prôner le repli sur soi, le projet cherchait à mettre en relation différentes communes se soutenant les unes les autres, créant ainsi une puissance socialiste effective à côté de l'État et du capital, de façon à rendre progressivement ces deux derniers superflus.

C'est sur ce dernier point que Landauer se distingue le plus radicalement de ceux qu'il

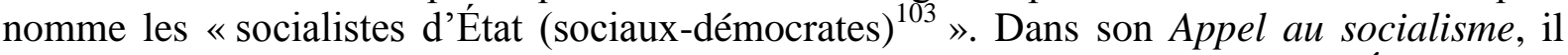
souligne cette contradiction apparente de la doctrine marxiste : «Capitalisme et État doivent converger, et c'est alors - nous dirions : et c'est alors qu'existe le capitalisme d'État ; mais ces marxistes disent: et c'est alors qu'existe le socialisme ${ }^{104}$.» Le constat de cette incohérence conduira Landauer à proposer une autre direction, directement orientée vers la réalisation effective du socialisme, ici et maintenant, dans des communes dont la mise en relation devrait conduire à la constitution d'une "société de sociétés ${ }^{105}$ »: un système fédératif ne reposant ni sur le centralisme de l'État, ni sur la domination du capital.

Nous ne nous intéressons pas au parti, pour les dirigeants duquel le socialisme ne constitue qu'un moyen en vue du pouvoir politique [...]. Nous trouvons que le socialisme n'a pas pire ennemi que toute forme de pouvoir politique ; que le socialisme a précisément pour tâche d'établir un ordre social et juridique qui prenne la relève de tout pouvoir politique ${ }^{106}$.

L'évolution historique du SPD et la constitution d'une doctrine unifiée et hégémonique en son sein ont rendu inconcevable cette abolition du pouvoir politique. Mais pour Landauer, cette puissance de l'appareil politique social-démocrate est un effet direct de l'étroitesse de sa conception du socialisme : «encore et toujours, la social-démocratie ne connaît nul chemin qui mène au socialisme, et c'est pourquoi elle s'adonne à la politique de parti ${ }^{107}$. Avec la progressive ascension vers le pouvoir du SPD, ceux qui redoutaient de devenir un «parti de gouvernement» et plus un «parti révolutionnaire» virent ainsi leurs craintes confirmées. Dans deux articles intitulés «Le parti », Landauer ironise sur cette inconsistance du marxisme de la Deuxième internationale, tiraillé entre une doctrine révolutionnaire et une pratique parlementaire d'accession au pouvoir ${ }^{108}$. Les «réformistes » qui, avec Eduard Bernstein,

\footnotetext{
${ }^{101}$ Landauer Gustav, «Que faire pour commencer? » (1909), Angaut Jean-Christophe et Lucet Anatole (trad.), in Appel au socialisme, op. cit., p. 216.

${ }^{102}$ Landauer Gustav, «Les douze articles de l'Alliance socialiste » (1908), art. cit., p. 199.

${ }^{103}$ Landauer Gustav, «Henry George und der sozialdemokratische Agrarprogrammentwurf », Der Sozialist, V, $\mathrm{n}^{\mathrm{o}} 1,17$ août 1895, GLAS 3.1, p. 61.

${ }^{104}$ Landauer Gustav, Appel au socialisme, op. cit., p. 85.

${ }^{105}$ Landauer Gustav, La révolution (1907), Manale Margaret et Janover Louis (trad.), Arles, Sulliver, 2006, pp. $46-47$.

${ }^{106}$ Landauer Gustav, « August Bebel », Der Sozialist, V, no 15, 15 août 1913, GLAS 3.2, p. 209.

${ }^{107}$ Landauer Gustav, «Aus der Zeit. In Sachen der Konsumgenossenschaften », Der Sozialist, II, n ${ }^{\mathrm{o}} 19$, $1^{\mathrm{er}}$ octobre 1910, GLAS 3.1, p. 237.

${ }^{108}$ Landauer Gustav, «Die Partei », Der Sozialist, I, no 17, 15 octobre 1909, GLAS 3.2, pp. 152-153. Les ressemblances du propos de Landauer avec l'analyse formulée par Eduard Bernstein et les partisans du mouvement réformiste s'expliquent, selon l'anarchiste, par le fait que ceux-ci auraient emprunté l'essentiel de leur critique «à l'anarchisme, à Eugen Dühring et à d'autres socialistes indépendants » (Appel au socialisme, op.cit., p. 139). Dès 1893, Landauer avait critiqué les faux-semblants de l'alternative entre réforme et révolution, mettant ainsi en lumière les promesses non tenues de la phraséologie révolutionnaire marxiste : «An den Züricher Kongress! », Der Sozialist, III, n ${ }^{0} 32,5$ août 1893, GLAS 2, p. 153. Cf. également Ein Weg zur Befreiung der Arbeiter-Klasse, op. cit., GLAS 14, pp. 68-69.
} 
prêchent l'abandon de la rhétorique révolutionnaire, ont au moins l'avantage de la clarté aux yeux de Landauer :

Bien, répondent les révisionnistes avec une grande tranquillité d'esprit, il est grand temps que nous renoncions enfin à ces grandes tournures. Tous ensemble, nous sommes un parti ouvrier démocratique et parlementaire : renonçons enfin à cet écartèlement tout à fait insupportable, qui épuise les nerfs ! Clarifions les choses!

C'est pour cela que les radicaux ne sont pas sympathiques : parce qu'ils n'osent pas la clarté. Ils n'en veulent pas, parce que tout leur socialisme coïncide avec leur organisation de parti. Ils ne sont rien s'ils n'ont pas leur ribambelle d'adhérents, et ils n'ont ces masses que par la politique parlementaire et électorale qu'ils mènent ${ }^{109}$.

Finalement, ni la voie résolument parlementaire des révisionnistes, ni les hésitations entre la théorie et la pratique dans la direction du parti, ni même les vues plus radicales et indépendantes sur l'aile gauche du parti n'emportent l'adhésion de Landauer ${ }^{110}$. Ce dernier continue bien plutôt de prôner la multiplication des expérimentations par des pionniers, œuvrant à la réalisation de nouvelles formes de vie commune sans redouter les revers de fortune. En assumant la place de l'échec dans les tentatives de changement social, Landauer se distingue encore radicalement du marxisme de son temps dont il écrit qu'il «fait toujours référence, moqueur et triomphal, à des défaites et à de vaines tentatives, et [qu']il a une telle peur puérile de l'échec. Il n'est rien contre quoi il affiche plus de mépris que ce qu'il appelle expériences ou fondations avortées ${ }^{111} »$. Contre cette frilosité du parti marxiste allemand, enfermé dans un attentisme stérile sous la Deuxième internationale, Landauer défend une perspective socialiste résolument étrangère au jeu des institutions politiques : "Nous devons donner l'exemple des précurseurs ${ }^{112}$. »

\section{CONCLUSION}

Les observateurs de la violente polémique qui opposa Gustav Landauer à la socialdémocratie allemande dans les pages du Vorwärts au tournant du $\mathrm{XX}^{\mathrm{e}}$ siècle auraient tôt fait de prendre fait et cause pour l'un ou l'autre des antagonistes, tant l'absence de nuances prédomine dans cet échange. Mais la virulence du propos masque une controverse de fond sur deux conceptions opposées du socialisme.

\footnotetext{
${ }^{109}$ Landauer Gustav, «Die Partei », art. cit., GLAS 3.2, p. 154. C'est dans son ouvrage de 1899 intitulé Les présupposés du socialisme, traduit par l'anarchiste Alexandre Cohen sous le titre Socialisme théorique et socialdémocratie pratique (Paris, Stock, 1900), que Bernstein en appelle à cette clarification. Les réactions occasionnées par cette «querelle du réformisme » au sein de la social-démocratie (notamment celles de Rosa Luxemburg et de Karl Kautsky) sont consignées dans l'ouvrage de Papaioannou Kostas, Marx et les marxistes, Paris, Gallimard, 2001, pp. 244-258.

${ }^{110}$ Dans une lettre écrite au début de la révolution, Landauer qualifie Karl Liebknecht et Rosa Luxemburg de «purs centralistes comme Robespierre et les siens, dont l'aspiration est dénuée de tout contenu, mais ne concerne que le pouvoir » (lettre à Margarete Susman du 13 décembre 1918, reproduite dans Sein Lebensgang in Briefen, op. cit., vol. 2/2, p. 336). Il est intéressant que Landauer ait été qualifié de «spartakiste» dans le Vorwärts au moment de la révolution allemande («Wirrwarr in München. Die Münchner Truppen gegen die Terroristen-Diktatur », Vorwärts, 36, nº 117, 5 mars 1919, p. 2). Cette erreur indique qu'il n'était plus au cœur de l'attention du journal à cette époque ; cela souligne également l'amalgame entretenu par le parti entre toutes les tendances socialistes dissidentes.

${ }^{111}$ Landauer Gustav, Appel au socialisme, op. cit., p. 74.

112 Ibid., p. 182.
} 
La prétention du parti social-démocrate à détenir un monopole sur la définition même du socialisme est en grande partie à l'origine de cette polémique, et les convictions libertaires de Gustav Landauer se sont certainement trouvées renforcées par la brutale hégémonie doctrinaire des théoriciens et dirigeants marxistes. Le dénigrement de toute option dissidente transparaît ainsi dans des articles à l'ironie cinglante, à l'image d'un rapport sur le congrès socialiste de Zürich qui se conclut en ces termes :

Une fois que le président Lang s'en fut pris de nouveau aux [socialistes] indépendants et qu'il eut exhorté à l'union, il leva la séance [...] avec une ovation passionnée pour la social-démocratie, une et indivisible ${ }^{113}$.

L'analyse des apparitions de Gustav Landauer dans le Vorwärts a permis de déceler, dans les dits et les non-dits de cette controverse, la stratégie de délégitimation de l'anarchisme mise en œuvre par l'organe de presse du parti marxiste. La fréquence des références à l'auteur anarchiste-socialiste et la manière dont ses interventions furent traitées indiquent l'importance du rôle que lui prêta le Vorwärts dans sa tentative de dénigrer toute alternative socialiste. Présenté comme un agitateur de peu de poids, une simple figure littéraire ou un fanatique borné et ignorant de la doctrine socialiste, Landauer retourna le compliment à ses opposants dans des pages truculentes. Mais derrière cette façade discursive, la controverse entre le Vorwärts et Gustav Landauer est surtout révélatrice d'une série de désaccords structurels et fondamentaux sur la conception de l'histoire, de la société, de l'expérimentation et du changement social. Les quelques points abordés devraient être complétés, dans un écrit plus long, par une discussion sur la conception landauerienne de l'utopie, de la technique, du progrès, de la grève ou du communisme non-marxiste : autant d'aspects sur lesquels il dut prendre position contre le marxisme de son temps pour s'efforcer « de donner au socialisme une nouvelle direction ${ }^{114}$ ».

\footnotetext{
113 «Aus der Schweiz », art. cit., p. 6.

114 «Zahme Anarchisten », art. cit., p. 2.
} 